\title{
Injection rate of contrast medium affects diagnostic ability of dynamic contrast-enhanced magnetic resonance imaging for endometrial carcinoma: a prospective cohort study
}

\author{
Donghui $\mathrm{Xu}^{1}$, Aiping Jin ${ }^{1}$, Yongqian $\mathrm{Ge}^{1}$, Yanmei Zhang ${ }^{2}$ \\ ${ }^{1}$ Department of Radiology, Affiliated Hospital of Nantong University, Nantong, China; ${ }^{2}$ Department of Obstetrics and Gynecology, General \\ Hospital of PLA Eastern Theater Command, Nanjing, China \\ Contributions: (I) Conception and design: D Xu, Y Zhang; (II) Administrative support: Y Zhang; (III) Provision of study materials or patients: D Xu, A \\ Jin, Y Ge; (IV) Collection and assembly of data: D Xu, A Jin, Y Ge; (V) Data analysis and interpretation: D Xu; (VI) Manuscript writing: All authors; \\ (VII) Final approval of manuscript: All authors. \\ Correspondence to: Yanmei Zhang. Department of Obstetrics and Gynecology, General Hospital of Eastern Theater Command, 305 Zhongshan East \\ Road, Nanjing 210002, China. Email: liumingyier@foxmail.com.
}

Background: Dynamic contrast enhanced magnetic resonance imaging (DCE-MRI) can obtain semi-
quantitative or quantitative parameters of tumors by capturing the images before and after injection of
contrast medium. However, there has been no further research on the effect of flow rate of contrast medium
on image quality and parameter sensitivity of DCE-MRI in endometrial carcinoma (EC).

Methods: This was a prospective cohort study enrolling patients who were suspected of EC between January 2018 and June 2020. The baseline data of participants were collected. Post-surgical histological examination acted as the gold standard of EC diagnosis and some characteristics of tumors were recorded. We calculated 3 important parameters of DCE-MRI, including volume transfer constant $\left(\mathrm{K}^{\text {trans }}\right)$, flux rate constant $\left(\mathrm{K}_{\mathrm{ep}}\right)$, and extravascular extracellular volume fraction $\left(\mathrm{V}_{\mathrm{e}}\right)$, according to the MRI system. The image quality in DCE-MRI imaging was evaluated according to contrast, resolution, artifact, signal-to-noise ratio, and scanning time. To evaluate the diagnostic ability of DCE-MRI with different injection rate, receiver operating characteristic (ROC) curve was generated and the area under curve (AUC) was calculated.

Results: According to the different injection rate of contrast medium, participants were divided into three groups, including 2, 3, and $4 \mathrm{~mL} / \mathrm{s}$ group. It was found that there were more grade $1 \mathrm{EC}$ in the $3 \mathrm{~mL} / \mathrm{s}$ group (52.4\%) than other two groups (34.3\% and $23.3 \%$, respectively), and the difference was significant $(\mathrm{P}=0.021)$. No other significant differences were found among all other variables. It was found that $\mathrm{K}^{\text {trans }}$ was much higher in the $4 \mathrm{~mL} / \mathrm{s}$ group than in other two groups $(\mathrm{P}<0.001)$. Also, $\mathrm{V}_{\mathrm{e}}$ was much higher in the $4 \mathrm{~mL} / \mathrm{s}$ group than in other two groups $(\mathrm{P}<0.001)$. However, no significant difference was found in $\mathrm{K}_{\mathrm{ep}}$ between three groups $(\mathrm{P}=0.633)$. Besides, the $4 \mathrm{~mL} / \mathrm{s}$ group had the highest quality of all three groups $(\mathrm{P}<0.001)$. The sensitivity, specificity, and accuracy were highest in $4 \mathrm{~mL} / \mathrm{s}$ group. The AUC in three groups were 0.822 , 0.832 , and 0.888 in the 2,3 , and $4 \mathrm{~mL} / \mathrm{s}$ group, respectively.

Conclusions: The DCE-MRI measurement is useful for the diagnosis of EC, and faster injection rate may be beneficial to improve diagnostic accuracy and image quality.

Keywords: Injection rate; contrast medium; dynamic contrast-enhanced magnetic resonance imaging; endometrial carcinoma (EC); diagnostic ability

Submitted Jun 04, 2021. Accepted for publication Aug 10, 2021.

doi: 10.21037 /gs-21-374

View this article at: https://dx.doi.org/10.21037/gs-21-374 


\section{Introduction}

Endometrial carcinoma (EC) is 1 of the most common gynecological malignant tumors and the incidence of EC has been increasing rapidly in China in recent decades (1-3). The most common surgical treatment for EC includes total hysterectomy, bilateral ovariectomy and salpingectomy, and pelvic lymph node dissection, which undoubtedly seriously affect the quality of life of patients. At present, a large number of studies have shown that patients with early stage EC can retain their fertility and achieve a long-term survival rate through minimally invasive surgical treatment and antiestrogen therapy (4-6). Therefore, accurate preoperative prediction of pathological staging and grading of EC is inarguably beneficial to the selection of treatment program, and improving the quality of life.

At present, surgery or invasive biopsy is used to assess the pathological staging and grading of EC; however, they cannot fully describe the heterogeneity of tumor $(7,8)$. Quantitative dynamic contrast enhanced magnetic resonance imaging (DCE-MRI), a continuous, repetitive, and fast imaging technology, can obtain semi-quantitative or quantitative parameters of tumors by capturing the images before and after injection of contrast medium (9). Compared with conventional magnetic resonance imaging (MRI), DCE-MRI can not only provide the morphological characteristics of tumors, but also indicate the physiological changes of tumors $(10,11)$.

There are some factors that may affect the imaging quality of DCE-MRI, 1 of which is the injection rate of contrast medium. On the one hand, low injection rate may lead to insufficient contrast medium concentration in the microcirculation of the target tissues and result in unclear images of tissues. On the other hand, high injection rate has a tendency to result in contrast medium extravasation and cause patient discomfort. Due to the varied requirements of different tissues and organs for scanning conditions, the injection rate of contrast medium used in DCE-MRI of different tissues and organs may also be different. It has been reported that intravenous administration of gadolinium ethoxybenzyldiethylenetriaminepentaacetic acid (Gd-EOB-DTPA) with an injection rate of $2 \mathrm{~mL} / \mathrm{s}$ is suitable for the detection of hepatocellular carcinoma $(12,13)$. In pediatric patients, a low peripheral intravenous gadoteric acid injection rate of $1 \mathrm{~mL} / \mathrm{s}$ may be better than a higher injection rate in differentiating the grades of brain tumor (14). Thieme et al. reported in their study that an injection rate of
$2 \mathrm{~mL} / \mathrm{s}$ could be of great significance in the preoperative evaluation of EC biological characteristics (15). Nevertheless, there has been no further study on the effect of flow rate of contrast medium on image quality and parameter sensitivity of DCE-MRI in EC.

This study was designed to evaluate the effects of different flow rates of contrast medium on the diagnostic ability of DCE-MRI for EC. It aimed to determine the optimal flow rate of DCE-MRI contrast medium, further predict the biological characteristics of EC, and improve postoperative prognosis and quality of life of EC patients. We present the following article in accordance with the STARD reporting checklist (available at https://dx.doi. org/10.21037/gs-21-374).

\section{Methods}

\section{Study design}

This study was a prospective non-randomized cohort study performed in a single center, the Affiliated Hospital of Nantong University, between January 2018 and June 2020. All procedures performed in this study involving human participants were in accordance with the Declaration of Helsinki (as revised in 2013). The study was approved by the Ethics Committee of the Affiliated Hospital of Nantong University (No. 2017015) and informed consent was taken from all the patients.

\section{Study population}

Patients who underwent examination by DCE-MRI for EC during the study period were enrolled in this study. The inclusion criteria were as follows: (I) patients who were suspected of having EC; (II) DCE-MRI examination was necessary for diagnosis; (III) surgical treatment was performed within 2 weeks after DCE-MRI examination. The exclusion criteria were as follows: (I) contraindications for DCE-MRI examination (including contrast medium allergy); (II) Intolerance of DCE-MRI; (III) refusal to receive surgical treatment within 2 weeks following DCEMRI; (IV) patients received radiotherapy and chemotherapy before examination. After patients had been enrolled in this study, some their baseline data were recorded, including their age, body mass index (BMI), menopausal status, and the level of cancer antigen 125 (CA125). The histological examination following surgery acted as the gold standard of EC diagnosis and some characteristics of tumors were 
recorded after surgery, including tumor grade, tumor size, tumor histological type, and myometrial invasion.

\section{DCE-MRI}

In this study, DCE-MRI was performed using a 3-Tesla system (GE Medical Systems, Waukesha, WI, USA) equipped with an 8-channel coil. The MRI protocol included standard T1-weighted imaging, T2-weighted imaging, and diffusion-weighted imaging. In order to obtain DCE-MRI images, $0.1 \mathrm{mmol} / \mathrm{kg}$ gadolinium-based contrast medium (Omniscan, Oslo, Norway) was intravenously given to patients using an automatic power injector. The injection rate was set at 2,3 , or $4 \mathrm{~mL} / \mathrm{s}$ according to the study protocol. Then, $16 \mathrm{~mL}$ normal saline was injected for flushing. Images were obtained at 0, 15, 30, 60, 90, 180 , and $300 \mathrm{~s}$ after the injection of contrast medium. The diagnosis of EC was confirmed independently by two skilled investigators.

\section{Image analysis}

We calculated three important parameters of DCE-MRI, including volume transfer constant $\left(\mathrm{K}^{\text {trans }}\right)$, flux rate constant $\left(\mathrm{K}_{\mathrm{ep}}\right)$, and extravascular extracellular volume fraction $\left(\mathrm{V}_{\mathrm{e}}\right)$, according to the MRI system. Moreover, the iliac artery and tumor parenchyma were selected as regions of interest (ROI) in the DCE-MRI images. The image quality in DCE-MRI imaging with different injection rates were compared according to the 2 ROI. The evaluation index included contrast, resolution, artifact, signal-to-noise ratio, and scanning time. Each item was scored from 0 to 2 points. Total score of $0-3$ points was defined as poor quality, 4-6 points as medium quality, and 7-10 points as high quality. In addition, the image quality would be reduced by a grade if there was extravasation of contrast medium or the participant was extremely uncomfortable during the examination.

\section{Statistical analysis}

All statistical analysis was performed using SPSS version 18.0 (IBM Corp., Armonk, NY, USA). According to the different injection rate of contrast medium, participants were divided into three groups, including 2, 3, and $4 \mathrm{~mL} / \mathrm{s}$ group. The sensitivity, specificity, positive predictive value (PPV), negative predictive value (NPV), and accuracy of DCE-MRI in the diagnosis of CE were obtained by comparing the results of imaging examination and histological examination. Their continuous variables were expressed as mean with standard deviation (SD) and categorical variables were expressed as number with percentage. Analysis of variance analysis (ANOVA) was used to compare the differences of continuous variables in baseline data, DCE-MRI parameters, and image quality between the three groups. The chi-square $\left(\chi^{2}\right)$ test was then used to compare the differences of categorical variables in baseline data and tumor characteristics between the three groups. To evaluate the diagnostic ability of DCE-MRI with different injection rates, receiver operating characteristic (ROC) curve was generated and the area under curve (AUC) was calculated. A P value less than 0.05 was considered statistically significant in this study.

\section{Results}

A total of 369 patients who were suspected of EC were admitted to our hospital during the study period. According to the inclusion and exclusion criteria, 122 patients were excluded from our study (Figure 1). Finally, 247 patients were enrolled in our study and their baseline data are displayed in Table 1. The mean age of participants was $62.0 \pm 10.9$ years and mean BMI was 26.2 \pm 6.7 . Menopausal status was also recorded, including 91 premenopausal participants and 156 postmenopausal participants. The level of CA125 was measured in all enrolled patients, which may be related to the incidence of EC. There were 60 participants $(24.3 \%)$ with a level of CA125 higher than $30 \mathrm{IU} / \mathrm{L}$.

The participants received surgical treatment within 2 weeks of DCE-MRI examination. Histological examination was used to confirm the characteristics of the tumors, and 39 of them were found to be benign, as shown in Table 2. Among the remaining 208 tumors, 78 were defined as grade 1, 109 as grade 2, and 21 tumors as grade 3 . The size of most tumors in participants (77.9\%) were larger than $2 \mathrm{~cm}$. A total of 185 tumors were found to be endometrioid carcinomas, followed by 9 serous or clear cell carcinomas, and 14 other carcinomas. The level of myometrial invasion was recorded, with a level of more than $50 \%$ exhibited in 49 tumors (23.6\%).

Next participants were divided into three groups according to different injection rate, including 120 participants in the $2 \mathrm{~mL} / \mathrm{s}$ group, 74 in the $3 \mathrm{~mL} / \mathrm{s}$ group, and 53 in the $4 \mathrm{~mL} / \mathrm{s}$ group. Baseline data and tumor characteristics are listed in Table 3. Comparison of these 


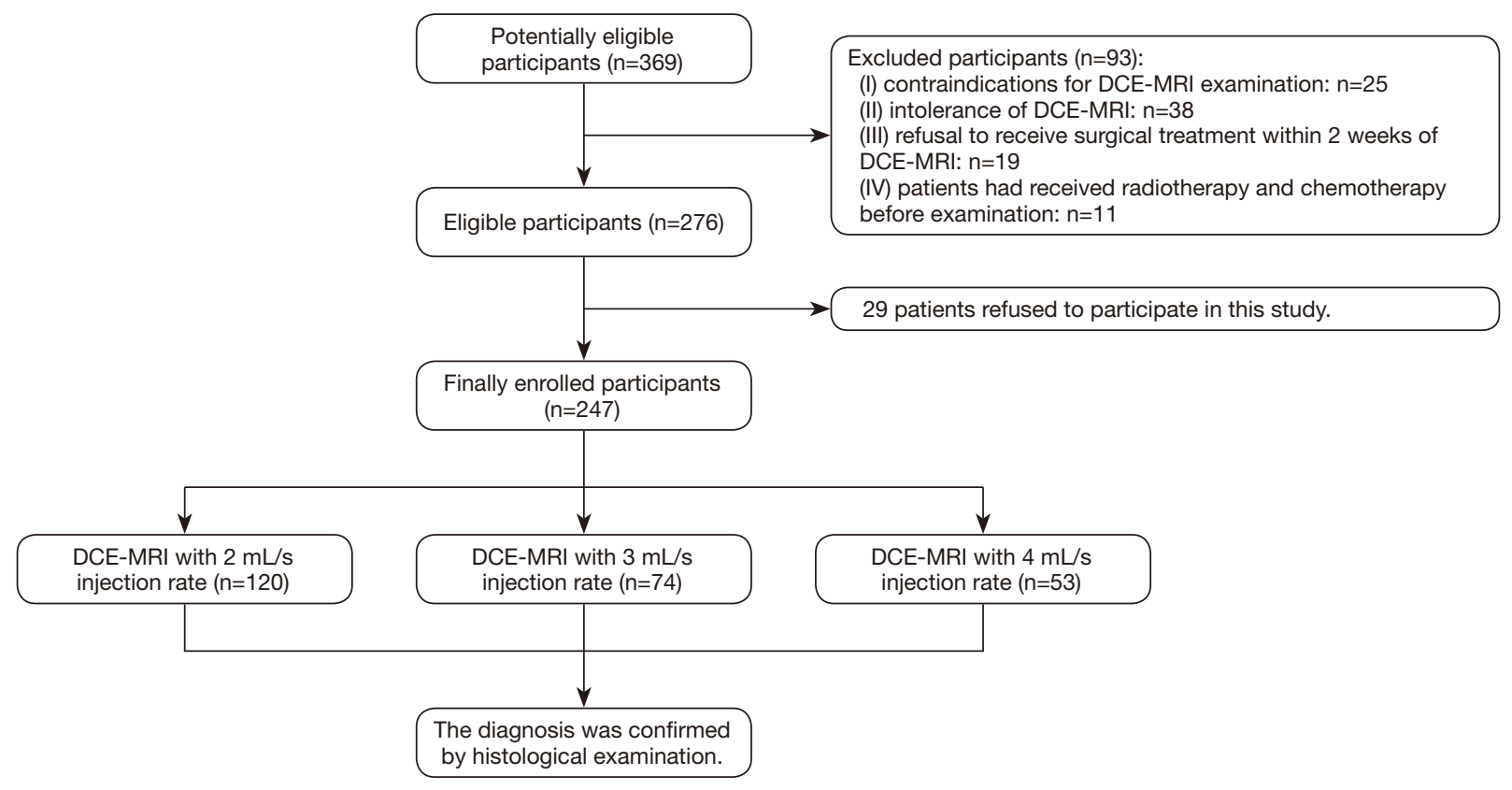

Figure 1 Flow chart of this study.

Table 1 Participant baseline data

\begin{tabular}{lc}
\hline Variables & Value \\
\hline Number & 247 \\
Age, year & $62.0 \pm 10.9$ \\
BMl, kg/m ${ }^{2}$ & $26.2 \pm 6.7$ \\
Menopausal status, $\mathrm{n}(\%)$ & \\
Premenopausal & $91(36.8)$ \\
Postmenopausal & $156(63.2)$ \\
Level of CA125 (IU/L), $\mathrm{n}(\%)$ & \\
$<30$ & $60(24.3)$ \\
$\geq 30$ & $187(75.7)$ \\
\hline
\end{tabular}

BMI, body mass index.

data between the three groups revealed that there were more grade $1 \mathrm{EC}$ in the $3 \mathrm{~mL} / \mathrm{s}$ group (52.4\%) than the other two groups ( $34.3 \%$ and $23.3 \%$, respectively), and the difference was significant $(\mathrm{P}=0.021)$. No other significant difference was found among all other variables.

The MRI parameters and image quality in all three
Table 2 Tumor characteristics of participants

\begin{tabular}{lc}
\hline Variables & Value \\
\hline Number & 247 \\
Tumor/benign lesion & $208 / 39$ \\
Tumor grade, $\mathrm{n}(\%)$ & \\
1 & $78(37.5)$ \\
2 & $109(52.4)$ \\
3 & $21(10.1)$ \\
Tumor size (cm), $\mathrm{n}(\%)$ & \\
$\leq 2$ & $46(22.1)$ \\
$>2$ & $162(77.9)$ \\
Tumor histological type, $\mathrm{n}(\%)$ & $185(88.9)$ \\
Endometrioid & $9(4.3)$ \\
Serous/clear cell & $14(6.7)$ \\
Other/mixed & $159(76.4)$ \\
Myometrial invasion, $\mathrm{n}(\%)$ & $49(23.6)$ \\
$<50 \%$ &
\end{tabular}


Table 3 Comparisons of baseline data and tumor characteristics between three groups of different injection rate

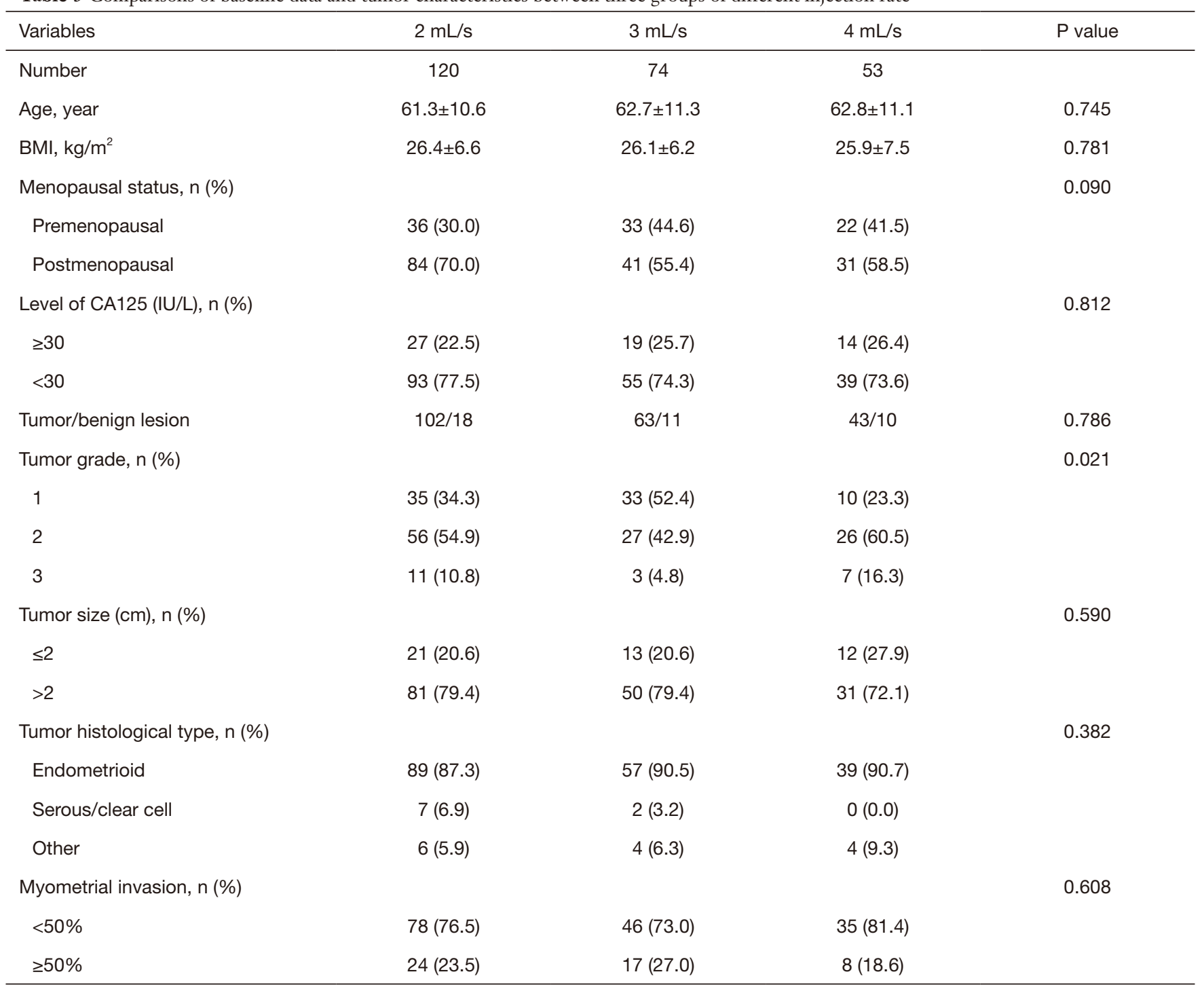

BMI, body mass index.

groups are displayed in Table 4. It was found that the value of $K^{\text {trans }}$ was much higher in the $4 \mathrm{~mL} / \mathrm{s}$ group than in the other two groups $(0.51 \pm 0.11 / \mathrm{min}$ in $4 \mathrm{~mL} / \mathrm{s}$ group vs. $0.41 \pm 0.09 / \mathrm{min}$ in $2 \mathrm{~mL} / \mathrm{s}$ group and $0.43 \pm 0.12 / \mathrm{min}$ in $3 \mathrm{~mL} / \mathrm{s}$ group, $\mathrm{P}<0.001)$. Also, the value of $\mathrm{V}_{\mathrm{e}}$ was much higher in the $4 \mathrm{~mL} / \mathrm{s}$ group than in the other two groups $(0.67 \pm 0.31$ in $4 \mathrm{~mL} / \mathrm{s}$ group $v s .0 .55 \pm 0.22$ in $2 \mathrm{~mL} / \mathrm{s}$ group and $0.60 \pm 0.32$ in $3 \mathrm{~mL} / \mathrm{s}$ group, $\mathrm{P}<0.001)$. However, no significant difference was found in the value of $\mathrm{K}_{\text {ep }}$ between the three groups. Then, image quality was evaluated according to 5 evaluation indexes, indicating that the $4 \mathrm{~mL} / \mathrm{s}$ group had the highest quality in all groups $(8.0 \pm 1.8$ in $4 \mathrm{~mL} / \mathrm{s}$ group versus $6.6 \pm 1.7$ in $2 \mathrm{~mL} / \mathrm{s}$ group and $7.2 \pm 1.9$ in $3 \mathrm{~mL} / \mathrm{s}$ group, $\mathrm{P}<0.001$ ).

Diagnostic ability of DCE-MRI with different injection rate is summarized in Table 5. It was found that sensitivity, specificity, and accuracy were $92.2 \%, 72.2 \%$, and $89.2 \%$, in the $2 \mathrm{~mL} / \mathrm{s}$ group; $93.7 \%, 72.3 \%$, and $90.5 \%$ in the $3 \mathrm{~mL} / \mathrm{s}$ group, and $97.7 \%, 80 \%$, and $94.3 \%$ in the $4 \mathrm{~mL} / \mathrm{s}$ group, respectively. The AUC in the three groups are shown in Figure 2, and the values were $0.822,0.832$, and 0.888 in the $2 \mathrm{~mL} / \mathrm{s}, 3 \mathrm{~mL} / \mathrm{s}$, and $4 \mathrm{~mL} / \mathrm{s}$ group, respectively. 
Table 4 Comparisons of MRI parameters and image quality between three groups

\begin{tabular}{lcccc}
\hline Variables & $2 \mathrm{~mL} / \mathrm{s}$ & $3 \mathrm{~mL} / \mathrm{s}$ & $4 \mathrm{~mL} / \mathrm{s}$ & $\mathrm{P}$ value \\
\hline $\mathrm{K}^{\text {trans }}, \mathrm{min}^{-1}$ & $0.41 \pm 0.09$ & $0.43 \pm 0.12$ & $0.51 \pm 0.11$ & $<0.001$ \\
$\mathrm{~K}_{\mathrm{ep}}, \mathrm{min}^{-1}$ & $0.79 \pm 0.18$ & $0.75 \pm 0.19$ & $0.79 \pm 0.2$ & 0.633 \\
$\mathrm{~V}_{\mathrm{e}}$ & $0.55 \pm 0.22$ & $0.60 \pm 0.32$ & $0.67 \pm 0.31$ & $<0.001$ \\
Image quality & $6.6 \pm 1.7$ & $7.2 \pm 1.9$ & $8.0 \pm 1.8$ & $<0.001$ \\
\hline
\end{tabular}

Table 5 Performance of DCE-MRI with different injection rates for the diagnosis of EC

\begin{tabular}{lccc}
\hline Variables & $2 \mathrm{~mL} / \mathrm{s}$ & $3 \mathrm{~mL} / \mathrm{s}$ & $4 \mathrm{~mL} / \mathrm{s}$ \\
\hline Sensitivity (\%) & 92.2 & 93.7 & 97.7 \\
Specificity (\%) & 72.2 & 72.3 & 80 \\
Positive predictive value (\%) & 94.9 & 95.2 & 95.5 \\
Negative predictive value (\%) & 61.9 & 66.7 & 88.9 \\
Accuracy (\%) & 89.2 & 90.5 & 94.3 \\
\hline
\end{tabular}

DCE-MRI, dynamic contrast enhanced magnetic resonance imaging; EC, endometrial carcinoma.
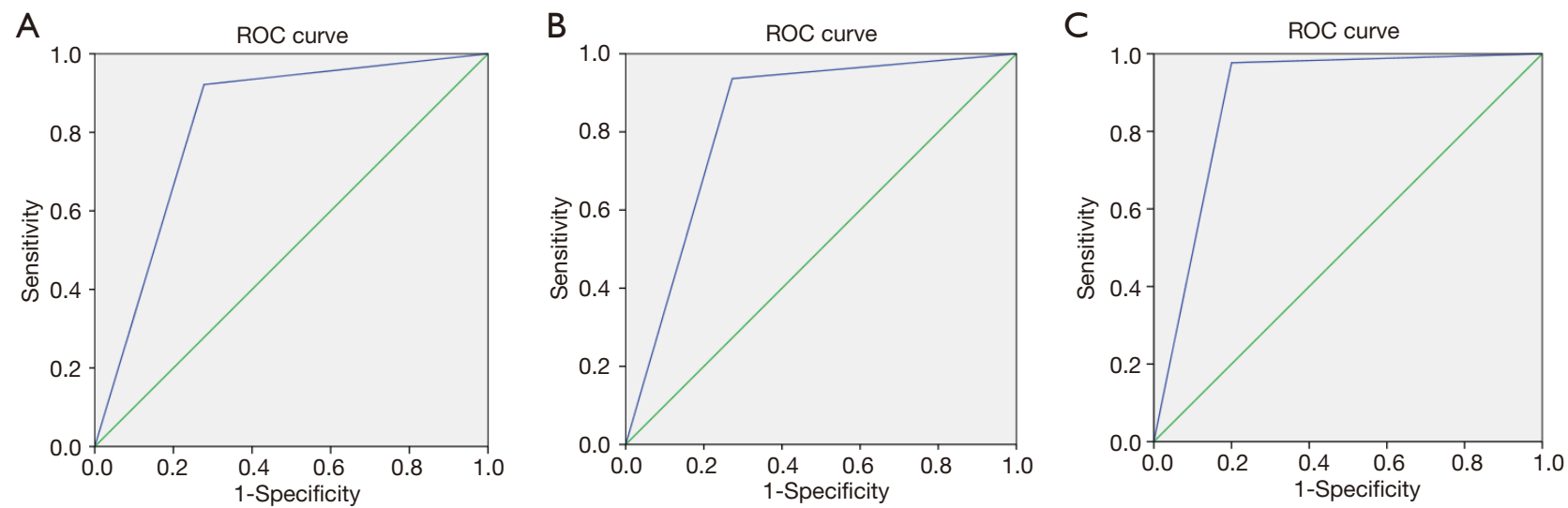

Figure 2 ROC curves of three groups. (A) $2 \mathrm{~mL} / \mathrm{s}$ group; (B) $3 \mathrm{~mL} / \mathrm{s}$ group; (C) $4 \mathrm{~mL} / \mathrm{s}$ group.

\section{Discussion}

To our knowledge, this was the first study to investigate the effect of different injection rates of contrast medium in DCE-MRI for EC. Totally, 247 patients suspected of EC were enrolled in our study and 208 participants were confirmed as EC. The results indicated that an injection rate of $4 \mathrm{~mL} / \mathrm{s}$ could provide the best image quality in DCE-MRI. Also, in the diagnosis of EC, the injection rate of $4 \mathrm{~mL} / \mathrm{s}$ can achieve the highest sensitivity, specificity, and accuracy of diagnosis. Our study provides some evidence for the management of the injection rate of contrast medium in
DEC-MRI during the diagnosis of EC. However, there are still some points worthy of discussion.

The three important MRI parameters, $\mathrm{K}^{\text {trans }}, \mathrm{K}_{\mathrm{ep}}$, and $\mathrm{V}_{\mathrm{e}}$, play important roles in the diagnosis of lesions. Usually, the values of $K^{\text {trans }}$ and $V_{e}$ are much higher in tumor tissue than in normal tissue. Chen et al. found in their study that the values of $\mathrm{K}^{\text {trans }}$ and $\mathrm{V}_{\mathrm{e}}$ in normal tissue differed significantly from those of tumor tissue (16). Besides, they were also significantly higher in metastatic nodes than in primary tumors (16). Conversely, according to a previous study the values of $K^{\text {trans }}$ and $V_{e}$ would not likely to be significantly 
different between tumors of high and low grades (17). The values of $\mathrm{K}^{\text {trans }}$ and $\mathrm{V}_{\mathrm{e}}$ in our study were much higher than those of the other two groups $(\mathrm{P}<0.001)$, which may facilitate distinguishing tumor tissue from normal tissue and improve the accuracy of diagnosis. However, the value of $\mathrm{K}_{\mathrm{ep}}$ may also be different between tumor tissue and normal tissue $(18,19)$. The 3 parameters have been widely used in recent years. It has been reported that the values of $\mathrm{K}^{\text {trans }}$, $\mathrm{K}_{\mathrm{ep}}$, and $\mathrm{V}_{\mathrm{e}}$ could be helpful in predicting the response of neoadjuvant chemotherapy in several types of tumors $(20,21)$, and we can now extrapolate their application to predicting the treatment response of EC.

We found that sensitivity, specificity, and accuracy were $92.2 \%, 72.2 \%$, and $89.2 \%$, respectively, in the $2 \mathrm{~mL} / \mathrm{s}$ group. The injection rate of $2 \mathrm{~mL} / \mathrm{s}$ is the most commonly used in DEC-MRI. In a previous study, it was reported that sensitivity, specificity, and accuracy were $92.3 \%, 86.4 \%$, and $88.6 \%$, respectively, when DEC-MRI was used for the diagnosis of EC (15). Our study had similar accuracy to the previous study. Zheng et al. applied the injection rate of $4 \mathrm{~mL} / \mathrm{s}$ in their study, obtaining a sensitivity of $100 \%$, specificity of $62.5 \%$, and accuracy of $86.4 \%$ (22); however, only 22 patients were enrolled in their study. Our study found that the sensitivity, specificity, and accuracy in the $4 \mathrm{~mL} / \mathrm{s}$ group were $97.7 \%$, $80 \%$, and $94.3 \%$, respectively. There was no significant difference between the $2 \mathrm{~mL} / \mathrm{s}$ and $3 \mathrm{~mL} / \mathrm{s}$ groups, while the diagnostic ability of DCEMRI was much higher in the $4 \mathrm{~mL} / \mathrm{s}$ than the other two groups. Accordingly, AUC in $4 \mathrm{~mL} / \mathrm{s}$ group was 0.888 , the highest of all groups. It is worth noting that when using the injection rate of $4 \mathrm{~mL} / \mathrm{s}$, attention should be paid to patient adverse reactions, because 3 patients of the $4 \mathrm{~mL} / \mathrm{s}$ group in our study experienced some discomfort during DCE-MRI examination.

There were some limitations in our study. The first is that our study enrolled relatively small samples in each of the three groups, especially in the $4 \mathrm{~mL} / \mathrm{s}$ group. In diagnostic trials, sample size is very important for the accuracy and stability of the results. There are more than 100 EC patients in our hospital every year, but the sample size was still insufficient after division into several groups. This limitation may be addressed through a multicenter prospective study. The second is that the grades of EC differed between the three groups, which may have affected the diagnostic ability of DEC-MRI. High grade tumors have larger volume or more extensive invasion, so the distribution of blood vessels in such tumors may be more extensive. The density of blood vessels is very important for the distribution of contrast medium (23). A randomized controlled study will be more beneficial for matching baseline data and tumor characteristics between groups.

\section{Conclusions}

To conclude, our study confirmed the diagnostic ability of DCE-MRI for EC, with a lowest accuracy rate of $89.2 \%$. Besides, no other study has investigated the effect of different injection rate on the diagnostic ability of DCEMRI for EC. As the first study focusing on injection rate of contrast medium, our research confirmed that faster injection rate may be beneficial to improve the diagnostic ability and image quality of DCE-MRI for EC. This study may provide some evidence for the usefulness of faster injection rate of contrast medium in the clinical setting.

\section{Acknowledgments}

Funding: This work was supported by the Scientific Research Project of Nantong Health Commission (MB2020078).

\section{Footnote}

Reporting Checklist: The authors have completed the STARD reporting checklist. Available at https://dx.doi. org/10.21037/gs-21-374

Data Sharing Statement: Available at https://dx.doi. org/10.21037/gs-21-374

Conflicts of Interest: All authors have completed the ICMJE uniform disclosure form (available at https://dx.doi. org/10.21037/gs-21-374). The authors have no conflicts of interest to declare.

Ethical Statement: The authors are accountable for all aspects of the work in ensuring that questions related to the accuracy or integrity of any part of the work are appropriately investigated and resolved. All procedures performed in this study involving human participants were in accordance with the Declaration of Helsinki (as revised in 2013). The study was approved by the Ethics Committee of the Affiliated Hospital of Nantong University (No. 2017015) and informed consent was taken from all the patients.

Open Access Statement: This is an Open Access article 
distributed in accordance with the Creative Commons Attribution-NonCommercial-NoDerivs 4.0 International License (CC BY-NC-ND 4.0), which permits the noncommercial replication and distribution of the article with the strict proviso that no changes or edits are made and the original work is properly cited (including links to both the formal publication through the relevant DOI and the license). See: https://creativecommons.org/licenses/by-nc-nd/4.0/.

\section{References}

1. Zhou JY, Zhang L, Wei LH, et al. Endometrial carcinomarelated genetic factors: application to research and clinical practice in China. BJOG 2016;123 Suppl 3:90-6.

2. Ding L, Ding Y, Mao XH, et al. Retrospective study of the prognostic significance of neutrophil-to-lymphocyte ratio for postsurgical outcomes of patients with endometrial carcinoma. Int J Gynaecol Obstet 2017;138:311-9.

3. $\mathrm{Li} \mathrm{Y,} \mathrm{He} \mathrm{Q,} \mathrm{Li} \mathrm{S,} \mathrm{et} \mathrm{al.} \mathrm{POLE} \mathrm{Mutation} \mathrm{Characteristics}$ in a Chinese Cohort with Endometrial Carcinoma. Onco Targets Ther 2020;13:7305-16.

4. Matsuo K, Matsuzaki S, Nusbaum DJ, et al. Association Between Adjuvant Therapy and Survival in Stage II-III Endometrial Cancer: Influence of Malignant Peritoneal Cytology. Ann Surg Oncol 2021. [Epub ahead of print]. doi: 10.1245/s10434-021-09900-4.

5. Cheng Y, Li X, Dai Y, et al. Identification of an immunerelated risk signature and nomogram predicting the overall survival in patients with endometrial cancer. J Gynecol Oncol 2021;32:e30.

6. Zhu L, Sun X, Bai W. Nomograms for Predicting CancerSpecific and Overall Survival Among Patients With Endometrial Carcinoma: A SEER Based Study. Front Oncol 2020;10:269.

7. Abdulfatah E, Wakeling E, Sakr S, et al. Molecular classification of endometrial carcinoma applied to endometrial biopsy specimens: Towards early personalized patient management. Gynecol Oncol 2019;154:467-74.

8. Goebel EA, St Laurent JD, Nucci MR, et al. Retrospective detection of isolated tumor cells by immunohistochemistry in sentinel lymph node biopsy performed for endometrial carcinoma: is there clinical significance? Int J Gynecol Cancer 2020;30:291-8.

9. Thomassin-Naggara I, Darai E, Cuenod CA, et al. Dynamic contrast-enhanced magnetic resonance imaging: a useful tool for characterizing ovarian epithelial tumors. JMRI 2008;28:111-20.

10. Gassenmaier S, Herrmann J, Nickel D, et al. Image
Quality Improvement of Dynamic Contrast-Enhanced Gradient Echo Magnetic Resonance Imaging by Iterative Denoising and Edge Enhancement. Invest Radiol 2021. [Epub ahead of print]. doi: 10.1097/ RLI.0000000000000761.

11. Onwuharine EN, Clark AJ. Comparison of double inversion recovery magnetic resonance imaging (DIRMRI) and dynamic contrast enhanced magnetic resonance imaging (DCE-MRI) in detection of prostate cancer: A pilot study. Radiography 2020;26:234-9.

12. Imbriaco M, De Luca S, Coppola M, et al. Diagnostic Accuracy of Gd-EOB-DTPA for Detection Hepatocellular Carcinoma (HCC): A Comparative Study with Dynamic Contrast Enhanced Magnetic Resonance Imaging (MRI) and Dynamic Contrast Enhanced Computed Tomography (CT). Pol J Radiol 2017;82:50-7.

13. Muñoz NM, Minhaj AA, Maldonado KL, et al. Comparison of dynamic contrast-enhanced magnetic resonance imaging and contrast-enhanced ultrasound for evaluation of the effects of sorafenib in a rat model of hepatocellular carcinoma. Magn Reson Imaging 2019;57:156-64.

14. Rochetams BB, Marechal B, Cottier JP, et al. T1-weighted dynamic contrast-enhanced brain magnetic resonance imaging: A preliminary study with low infusion rate in pediatric patients. Neuroradiol J 2017;30:429-36.

15. Thieme SF, Collettini F, Sehouli J, et al. Preoperative Evaluation of Myometrial Invasion in Endometrial Carcinoma: Prospective Intra-individual Comparison of Magnetic Resonance Volumetry, Diffusion-weighted and Dynamic Contrast-enhanced Magnetic Resonance Imaging. Anticancer Res 2018;38:4813-7.

16. Chen L, Ye Y, Chen H, et al. Dynamic Contrast-enhanced Magnetic Resonance Imaging for Differentiating Between Primary Tumor, Metastatic Node and Normal Tissue in Head and Neck Cancer. Curr Med Imaging Rev 2018;14:416-21.

17. Gupta PK, Saini J, Sahoo P, et al. Role of Dynamic Contrast-Enhanced Perfusion Magnetic Resonance Imaging in Grading of Pediatric Brain Tumors on 3T. Pediatr Neurosurg 2017;52:298-305.

18. Zhang Y, Yue B, Zhao X, et al. Benign or Malignant Characterization of Soft-Tissue Tumors by Using Semiquantitative and Quantitative Parameters of Dynamic Contrast-Enhanced Magnetic Resonance Imaging. Can Assoc Radiol J 2020;71:92-9.

19. Hou W, Li X, Pan H, et al. Dynamic contrast-enhanced magnetic resonance imaging for monitoring the anti- 
angiogenesis efficacy in a C6 glioma rat model. Acta Radiol 2020;61:973-82.

20. Guo W, Zhang Y, Luo D, et al. Dynamic contrastenhanced magnetic resonance imaging (DCE-MRI) for pretreatment prediction of neoadjuvant chemotherapy response in locally advanced hypopharyngeal cancer. Br J Radiol 2020;93:20200751.

21. Zheng D, Lai G, Chen Y, et al. Integrating dynamic contrast-enhanced magnetic resonance imaging and diffusion kurtosis imaging for neoadjuvant chemotherapy assessment of nasopharyngeal carcinoma. JMRI

2018;48:1208-16.

Cite this article as: $\mathrm{Xu} \mathrm{D}$, Jin A, Ge Y, Zhang Y. Injection rate of contrast medium affects diagnostic ability of dynamic contrast-enhanced magnetic resonance imaging for endometrial carcinoma: a prospective cohort study. Gland Surg 2021;10(8):24622470. doi: $10.21037 /$ gs-21-374
22. Zheng L, Zheng S, Yuan X, et al. Comparison of dynamic contrast-enhanced magnetic resonance imaging with T2-weighted imaging for preoperative staging of early endometrial carcinoma. Onco Targets Ther 2015;8:1743-51.

23. Gaudino S, Benenati M, Martucci M, et al. Investigating dynamic susceptibility contrast-enhanced perfusionweighted magnetic resonance imaging in posterior fossa tumors: differences and similarities with supratentorial tumors. Radiol Med 2020;125:416-22.

(English Language Editor: J. Jones) 